\section{Research Square}

\title{
Acetylshikonin Suppresses Diffuse Large B-Cell Lymphoma Cell Growth By Targeting The TOPK Signaling Pathway
}

Jieke Cui

The First Affiliated Hospital of Zhengzhou University

Rong Guo

The First Affiliated Hospital of Zhengzhou University

Yingjun Wang

The First Affiliated Hospital of Zhengzhou University

Yue Song

The First Affiliated Hospital of Zhengzhou University

Xuewen Song

Affiliated Cancer Hospital of Zhengzhou University

Chong Zeng

Shunde Hospital of Southern Medical University

Hongwen Li

The First Affiliated Hospital of Zhengzhou University

Jiwei Li ( $\nabla$ lijiwei.li@163.com )

The First Affiliated Hospital of Zhengzhou University

\section{Research}

Keywords: DLBCL, TOPK, acetylshikonin, cell growth

Posted Date: January 15th, 2021

DOI: https://doi.org/10.21203/rs.3.rs-146077/v1

License: (1) (1) This work is licensed under a Creative Commons Attribution 4.0 International License. Read Full License 


\section{Abstract}

Background: Diffuse large B-cell lymphoma (DLBCL) is one of the most common causes of cancer death worldwide, and responds badly to the existing treatment. Thus, identifying the novel therapeutic targets of DLBCL are urgent.

Methods and results: In this study, we found that the T-lymphokine-activated killer cell-originated protein kinase (TOPK) was highly expressed in DLBCL cells and tissues. The TOPK expression were analyzed by bioinformatics analysis, immunohistochemistry $(\mathrm{IHC})$ and western blot analysis. TOPK knockdown inhibited cell growth and induced apoptosis of DLBCL cells with MTS and flow cytometry. Further experiments demonstrated that acetylshikonin, the targeted compound of TOPK, could attenuate the cell growth and aggravate the cell apoptosis through TOPK/extra cellular signal-regulated kinase (ERK)-1/2 signaling using MTS, flow cytometry and western blot analysis. In addition, we demonstrated that TOPK overexpression significantly reduced the acetylshikonin effect on cell proliferation and apoptosis in U2932 and OCI-LY8 cells using MTS, flow cytometry and western blot analysis.

Conclusions: Taken together, the present study suggests that the targeted inhibition of TOPK by acetylshikonin may be a promising approach to the treatment of DLBCL.

\section{Background}

Diffuse large $B$ cell lymphoma (DLBCL) is the most common type of pathology in non-Hodgkin lymphoma (NHL), representing about $30 \%$ of all diagnosed $\mathrm{NHL}$ cases, adding rituximab to cyclophosphamide, doxorubicin, vincristine, and prednisone (R-CHOP) chemotherapy has been the standard treatment which significantly improved the survival rate of DLBCL patients[1-3]. However, there are still about $40 \%$ of patients with treatment failure, because of the biologic heterogeneity[4,5]. Thus, Investigating novel biomarkers are important for the treatment of DLBCL patients.

The lymphokine-activated killer T (T-LAK)-cell-originated protein kinase (TOPK) is a member of the serinethreonine kinases of the mitogen-activated protein kinase kinase (MAPKK) family and is highly expressed in many cancers, including adult T-cell leukemia/lymphoma (ATLL) and B cell lymphoma[6-10]. TOPK promotes cancer cell proliferation by its phosphorylation of ERKs[11, 12]. The knockdown of TOPK will activate cysteine proteases (caspase -3 and caspase -7 ) and finally kill the cancer cells $[13,14]$. In primary central nervous system lymphoma (PCNSL), TOPK can be a potent novel biomarker, and its overexpression is associated with poor prognosis of PCNSL[15]. However, the underlying role of TOPK in DLBCL remains unclear.

Studies have shown that the inhibition of TOPK could be the target in cancer chemoprevention and treatment[16]. There have been some studies to report the function of TOPK inhibitors, such as OTS964, which could inhibit glioma stem cells survival, but the surviving glioma stem cells start to regrow at last[17]. 3-DSC is found from the Caesalpinia sappan L. plant[18], which binds to the ATP binding pocket of TOPK, could inhibit colon cancer growth by targeting the TOPK signaling pathway in vitro[14]. 
Moreover, acetylshikonin, another TOPK inhibitor, reduces colorectal cancer cells proliferation and decreases the volume of patient-derived xenograft (PDX) tumors in mice[13]. These studies suggest that TOPK may be a promising target for anticancer therapy.

In this study, we report the crucial role of TOPK in DLBCL tumorigenesis, The konckdown of TOPK attenuated malignancy phenotypes of DLBCL cells, including cell proliferation and apoptosis induction. Furthermore, We demonstrate that acetylshikonin inhibits cell proliferation and induces cell apoptosis by through TOPK signaling pathway. These results suggest that TOPK may be a promising molecular target in DLBCL treatment.

\section{Materials And Methods}

\section{Reagents}

3-DSC (cat: JOT-10796) was purchased from Chengdu Pufei De Biotech Co., Ltd (Chengdu, China), acetylshikonin (cat: YRY134) was purchased from Chengdu Yirui Biotech Co., Ltd (Chengdu, China), Antibodies to detect total TOPK (cat: 4942), phosphorylated TOPK (cat: 4941), total ERKs (cat: 9102), phosphorylated ERKs (cat: 4370), caspase-3 (cat: 9662), caspase-7 (cat: 12827), cleaved caspase-3 (cat: 9664), cleaved caspase-7 (cat: 9491) and actin (cat: 3700) were purchased from Cell Signaling Technology (Beverly, MA, USA).

\section{Cell culture}

The human DLBCL cell lines (U2932, SUDHL-6 and OCI-Ly8) and normal B-cell line (WIL2S) were purchased from Type Culture Collection of the Chinese Academy of Sciences (Shanghai, China). The cells were cultured in RPMI 1640 medium with 10\% fetal bovine serum (FBS). WIL2S cells were maintained in iscove's modified medium with $10 \%$ human serum (NABI Biopharmaceuticals, Boca Raton, FL, USA) and 2 mM L-glutamine (Invitrogen, Carlsbad, CA, USA). All cells were cultured with penicillin/streptomycin antibiotic mixture, penicillin (100 U.ml-1), streptomycin $(100 \mu \mathrm{gml}-1)$ at $37^{\circ} \mathrm{C}$ in $5 \% \mathrm{CO}_{2}$.

\section{Bioinformatics analysis}

Gene Expression Profiling Interactive Analysis (GEPIA, http://gepia.cancer-pku.cn/) software was used to analyze the expression of TOPK in DLBCL. First, enter TOPK and select boxplots. Then, select DLBC under datasets selection (cancer name) and click plot button.

\section{Immunohistochemical (IHC) Staining}

DLBCL tissue array (cat: LY800b) was purchase from Alenabio biotechnology Co., Ltd, containing 62 cases of DLBCL samples and 20 cases of normal control tissue samples. IHC staining was detected followed by the previous protocol[19], using TOPK antibody (1:200).

\section{Western blot analysis}


The protein samples were separated with SDS-PAGE electrophoresis, then transferred to polyvinylidene difluoride (PVDF) membranes (Bio-Rad, Hercules, CA, USA). Blocking with defatted milk, incubating with the first antibodies (1:1000), including TOPK, PTOPK, ERK, PERK, caspase 3, caspase 7, cleaved caspase 3 , cleaved caspase 7 and actin, then incubating with the secondary antibodies. Images were captured with the Tanon-5200.

\section{Lentiviral infection}

Lentiviruses carrying TOPK-cDNA (accession number NM_018492.4), shTOPK.1 (ATTAGTGCATACAGAGAAGAGTT) and shTOPK.2 (GTCTGTGTCTTGCTATGGAAT) were from GenePharma Co. (Shanghai, China). For lentiviral infection, cells were infected with lentivirus (MOI=100), containing polybrene $(5 \mu \mathrm{g} / \mathrm{ml})$ for 1-3 days.

\section{Cell proliferation assay}

The cells were seeded $\left(2 \times 10^{3}\right.$ cells per well) in 96 -well plates. After incubation for 24,48 , or $72 \mathrm{~h}$, cell proliferation was measured by MTS assay kit.

\section{Apoptosis Detection}

Flow cytometry was performed to observe the cell apoptosis with annexin V-FITC apoptosis detection kit (beyotime, C1062S). Cells were collected and washed with cold PBS. Added FITC annexin V and PI, incubated for $15 \mathrm{~min}$ at room temperature in the dark. Then analyzed by flow cytometry within $1 \mathrm{~h}$.

\section{Statistical analysis}

Results were presented as the means \pm standard deviation (SD) of 3 independent experiments, each dosage or treatment was tested in triplicate. Statistical analyses were performed with GraphPad Prism 6. Student's $t$ test were used to analyze the significant differences when the $p$ value was less than 0.05 .

\section{Results}

\section{TOPK is expressed at high levels in DLBCL}

To explore the role of TOPK in DLBCL, we analyzed TOPK expression in DLBCL cancer specimens and normal controls in the dataset of GEPIA. As shown in Fig. 1a, TOPK expression was obviously upregulated in DLBCL cancer specimens than normal samples. This result was further confirmed by testing TOPK expression using IHC in the DLBCL tumor microarray that included 62 cancer tissues and 20 control tissues (Fig. 1b). Similarly, TOPK expression was upregulated in DLBCL cell lines (U2932, OClLY8, SUDHL-6) compared with normal B-cell line (WIL2S) in protein levels by western blot (Fig. 1C). These results suggest that TOPK upregulation may promote $D L B C L$ progression.

Silencing TOPK reduces the tumorigenic properties of DLBCL 
To gain the insight into the potential role of TOPK as an oncogene whose overexpression was associated with DLBCL tumorigenesis, we generated the TOPK knockdown cells infected with lentivirous, the knockdown efficiency was verified in OCl-LY8 cells by western blot (Fig. 2a). The cell proliferation assays were performed by MTS to evaluate the effect of TOPK knockdown. Results showed that TOPK knockdown inhibited proliferation in U2932 and OCI-LY8 cells (Fig. 2b-c).

Studies have shown that TOPK knockdown could induced cell apoptosis[20, 21]. Here, the annexin VFITC/PI double-staining assay results showed that TOPK knockdown increased apoptosis in U2932 and OCI-LY8 cells (Fig. 2d). We also observed that the cleavage of caspase-3 and caspase-7 were obviously enhanced by western blot (Fig. 2e, Figure. S1A). Overall, our data show that TOPK could modulate the cell proliferation and apoptosis in DLBCL.

\section{Acetylshikonin suppresses DLBCL cell growth by targeting TOPK signaling}

Previously studies have shown that acetylshikonin and 3-DSC could suppress colon cancer cell growth[13,14]. To study the effects of acetylshikonin and 3-DSC on DLBCL cell growth, we first assessed the effect of acetylshikonin and 3-DSC on the proliferation of normal B-cell line. Incubation of WIL2S cells with acetylshikonin 3-DSC $(0,5,10,20 \mu \mathrm{M})$ for 24,48 or $72 \mathrm{~h}$ did not show cytotoxicity up to $20 \mu \mathrm{M}$ at $72 \mathrm{~h}$ (Fig. 3a), crucially, acetylshikonin inhibited the cell growth of DLBCL cell lines (U2932 and OCI-LY8) in a time- and dose-dependent manner (Fig. 3c-d). However, 3-DSC have shown cytotoxicity of WIL2S and U2932 cells to $10 \mu \mathrm{M}$ at $24 \mathrm{~h}$ (Fig. 3b, 3e), but 3-DSC did not show cytotoxicity in OCI-LY8 for $10 \mu \mathrm{M}$ at $24 \mathrm{~h}$ (Fig. 3f), so we estimated that acetylshikonin was more sensitive in DLBCL. Next, the role of acetylshikonin on cell apoptosis were examined. Treatment with $10 \mu \mathrm{M}$ acetylshikonin at $24 \mathrm{~h}$ induced cell apoptosis in U2932 and OCI-LY8 cells (Fig. 4a). In addition, we determined whether acetylshikonin also affected TOPK signaling pathway, we determined the effects of acetylshikonin on the expression of phosphorylated TOPK (pTOPK), phosphorylated ERK (pERK). Treatment of U2932 and OCI-LY8 cells with $10 \mu \mathrm{M}$ acetylshikonin at $24 \mathrm{~h}$ decreased the levels of pTOPK and pERK, and the total levels of TOPK, ERK were unchanged as compared to DMSO controls (Fig. 4b, Figure. S1B).

\section{TOPK overexpression attenuates the effect of acetylshikonin.}

To further determine whether TOPK could mediate the acetylshikonin induced cell growth inhibition, we infected TOPK cDNA and treated with acetylshikonin in U2932 and OCI-LY8 cells, followed by analysis of cell proliferation and apoptosis. The TOPK overexpression efficiency was shown in U2932 and OCI-LY8 cells by western blot (Fig. 5d, Figure. S1C). Results showed that the inhibition of cell proliferation treated by acetylshikonin was partly rescued upon overexpression of TOPK (Fig. 5a-b) and the induction of cell apoptosis treated by acetylshikonin was partly inhibited by the overexpression of TOPK (Fig. 5c). The cleavage of caspase-3 and caspase-7 showed coincident results (Fig. 5d, Figure. S1C).

\section{Discussion}


Despite the improvements in the treatments of DLBCL, it is still associated with a high mortality rate[22], thus, exploring the novel factors that affects DLBCL progression is significant for targeted therapy. This study reports that TOPK has a key role in DLBCL tumorigenic properties. Acetylshikonin suppresses DLBCL cell growth by targeting the TOPK signaling pathway and may be a potential treatment strategy for DLBCL.

TOPK is an active form of MEK1 which can phosphorylate ERK1/2[12]. Studies have shown the correlation between TOPK expression and poor prognosis in several cancers, such as breast cancer[23], lung cancer[24], leukemia[25], burkitt's lymphoma[9]. The overexpression expression of TOPK is associated with the tumorigenesis[26]. Although TOPK plays important roles in multiple cancer development, its function in DLBCL remains unclear. This work evaluates the function of TOPK in DLBCL.

Our results indicated that TOPK showed high levels in DLBCL, both in DLBCL cell lines and tissues (Fig. 1). For the functional study, we demonstrated that TOPK knockdown dramatically inhibited cell proliferation and induced cell apoptosis (Fig. 2, Figure. S1A) in U2932 and OCI-LY8 cells. These results suggest that TOPK knockdown obviously attenuates malignancy phenotypes of DLBCL cells, and thus, searching the appropriate inhibitors which could target TOPK is pregnant for DLBCL treatment.

An ideal anti-cancer compound should lack cytotoxicity against normal cells. In this study, we choose two native compound (acetylshikonin and 3-DSC) which were extracted from plants and showed anti-cancer effects by target TOPK $[13,14]$. Our data indicated that acetylshikonin showed no cytotoxicity up to $20 \mu \mathrm{M}$ at $72 \mathrm{~h}$ (Fig. 3a), but 3-DSC showed cytotoxicity of normal B-cells (WIL2S) up to $10 \mu \mathrm{M}$ at $24 \mathrm{~h}$ (Fig. 3b). So we estimated that acetylshikonin was more suitable targeted compound for DLBCL. The observed anti-cancer effects of acetylshikonin was reported in human pancreatic panc-1 cancer cells and colon cancer cells[13,27]. In our studies, we demonstrated that acetylshikonin inhibited cell proliferation (Fig. 3c-d) and induced cell apoptosis (Fig. 4a) in U2932 and OCI-LY8 cells. Papers have shown that TOPK is a major target of acetylshikonin to inhibit proliferation and induce apoptosis, several signalling molecules, including ERK, is involved in TOPK signalling and acetylshikonin decreased the phosphorylation of TOPK, ERK[13]. Our results confirm that acetylshikonin could decrease the phosphorylation of TOPK, ERK in U2932 and OCI-LY8 cells (Fig. 4b, Figure. S1B). In addition, TOPK overexpression abolished the cell proliferation inhibition, cell apoptosis induction and the increase of cleaved caspase- 3 and caspase-7 that treatment with acetylshikonin, which further confirmed that TOPK could involved the acetylshikonin induced cell growth inhibition (Fig. 5, Figure. S1C).

\section{Conclusions}

In conclusion, the present study suggests that TOPK may mediate acetylshikonin induced cell growth inhibition in U2932 and OCI-LY8 cells. Acetylshikonin or its derivatives can be considered for the new target-based therapy for DLBCL and TOPK may be the candidate molecular target for DLBCL treatment.

\section{Abbreviations}


DLBCL: Diffuse large B-cell lymphoma; TOPK: T-lymphokine-activated killer cell-originated protein kinase; T-LAK: The lymphokine-activated killer T; IHC: immunohistochemistry; ERK: extra cellular signal-regulated kinase; NHL: non-Hodgkin lymphoma

\section{Declarations}

\section{Acknowledgments}

Not applicable

\section{Author Contributions}

J.W.L., J.K.C. and R.G. conceived and designed the experiments. J.K.C., R.G., Y.J.W., Y.S., X.W.S., C.Z. and H.W.L. performed the experiments. J.W.L., J.K.C. and R.G. analyzed the data. J.W.L. and C.Z. contributed the reagents. J.W.L. and J.K.C.wrote the paper. All authors read and approved the final manuscript.

\section{Funding}

This work was supported by the Medical Science and Technology Project of Henan Province (LHGJ20190038).

\section{Availability of data and materials}

The datasets supporting the conclusions of this article are included within the article and additional files.

\section{Ethics approval and consent to participate}

Not applicable

\section{Consent for publication}

Consent to publish has been obtained from the participants.

\section{Conflict of interest}

The authors declare that they have no conflicts of interest.

\section{References}

1. Tilly H, Gomes da Silva M, Vitolo U, Jack A, Meignan M, Lopez-Guillermo A, Walewski J, Andre M, Johnson PW, Pfreundschuh M et al: Diffuse large B-cell lymphoma (DLBCL): ESMO Clinical Practice Guidelines for diagnosis, treatment and follow-up. Annals of oncology : official journal of the European Society for Medical Oncology 2015, 26 Suppl 5:v116-125.

2. Wang Y, Zhang M, Xu H, Wang Y, Li Z, Chang Y, Wang X, Fu X, Zhou Z, Yang S et al: Discovery and validation of the tumor-suppressive function of long noncoding RNA PANDA in human diffuse large 
B-cell lymphoma through the inactivation of MAPK/ERK signaling pathway. Oncotarget 2017, 8(42):72182-72196.

3. Feugier P, Van Hoof A, Sebban C, Solal-Celigny P, Bouabdallah R, Ferme C, Christian B, Lepage E, Tilly $\mathrm{H}$, Morschhauser $\mathrm{F}$ et al: Long-term results of the R-CHOP study in the treatment of elderly patients with diffuse large B-cell lymphoma: a study by the Groupe d'Etude des Lymphomes de l'Adulte. Journal of clinical oncology : official journal of the American Society of Clinical Oncology 2005, 23(18):4117-4126.

4. Vercellino L, Cottereau AS, Casasnovas O, Tilly H, Feugier P, Chartier L, Fruchart C, Roulin L, Oberic L, Pica GM et al: High total metabolic tumor volume at baseline predicts survival independent of response to therapy. Blood 2020, 135(16):1396-1405.

5. Sehn LH, Gascoyne RD: Diffuse large B-cell lymphoma: optimizing outcome in the context of clinical and biologic heterogeneity. Blood 2015, 125(1):22-32.

6. Uchida E, Suwa S, Yoshimoto R, Watanabe K, Kasama T, Miura O, Fukuda T: TOPK is regulated by PP2A and BCR/ABL in leukemia and enhances cell proliferation. International journal of oncology 2019, 54(5):1785-1796.

7. Herbert KJ, Ashton TM, Prevo R, Pirovano G, Higgins GS: T-LAK cell-originated protein kinase (TOPK): an emerging target for cancer-specific therapeutics. Cell death \& disease 2018, 9(11):1089.

8. Park JH, Inoue H, Kato T, Zewde M, Miyamoto T, Matsuo Y, Salgia R, Nakamura Y: TOPK (T-LAK celloriginated protein kinase) inhibitor exhibits growth suppressive effect on small cell lung cancer. Cancer science 2017, 108(3):488-496.

9. Simons-Evelyn M, Bailey-Dell K, Toretsky JA, Ross DD, Fenton R, Kalvakolanu D, Rapoport AP: PBK/TOPK is a novel mitotic kinase which is upregulated in Burkitt's lymphoma and other highly proliferative malignant cells. Blood cells, molecules \& diseases 2001, 27(5):825-829.

10. Ishikawa C, Senba M, Mori N: Mitotic kinase PBK/TOPK as a therapeutic target for adult T-cell leukemia/lymphoma. International journal of oncology 2018, 53(2):801-814.

11. Kim DJ, Li Y, Reddy K, Lee MH, Kim MO, Cho YY, Lee SY, Kim JE, Bode AM, Dong Z: Novel TOPK inhibitor HI-TOPK-032 effectively suppresses colon cancer growth. Cancer research 2012, 72(12):3060-3068.

12. Zhu F, Zykova TA, Kang BS, Wang Z, Ebeling MC, Abe Y, Ma WY, Bode AM, Dong Z: Bidirectional signals transduced by TOPK-ERK interaction increase tumorigenesis of HCT116 colorectal cancer cells. Gastroenterology 2007, 133(1):219-231.

13. Zhao R, Choi BY, Wei L, Fredimoses M, Yin F, Fu X, Chen H, Liu K, Kundu JK, Dong Z et al: Acetylshikonin suppressed growth of colorectal tumour tissue and cells by inhibiting the intracellular kinase, T-lymphokine-activated killer cell-originated protein kinase. British journal of pharmacology 2020, 177(10):2303-2319.

14. Zhao R, Huang H, Choi BY, Liu X, Zhang M, Zhou S, Song M, Yin F, Chen H, Shim JH et al: Cell growth inhibition by 3-deoxysappanchalcone is mediated by directly targeting the TOPK signaling pathway 
in colon cancer. Phytomedicine : international journal of phytotherapy and phytopharmacology 2019, 61:152813.

15. Koh M, Hayakawa Y, Akai T, Hayashi T, Tomita T, Nagai S, Kuroda S: Novel biomarker, phosphorylated T-LAK cell-originated protein kinase (p-TOPK) can predict outcome in primary central nervous system lymphoma. Neuropathology : official journal of the Japanese Society of Neuropathology 2018, 38(3):228-236.

16. Matsuo Y, Park JH, Miyamoto T, Yamamoto S, Hisada S, Alachkar H, Nakamura Y: TOPK inhibitor induces complete tumor regression in xenograft models of human cancer through inhibition of cytokinesis. Science translational medicine 2014, 6(259):259ra145.

17. Sugimori M, Hayakawa $\mathrm{Y}$, Koh M, Hayashi T, Tamura R, Kuroda S: Targeting the T-Lak cell originated protein kinase by OTS964 shrinks the size of power-law coded heterogeneous glioma stem cell populations. Oncotarget 2018, 9(3):3043-3059.

18. Yodsaoue O, Cheenpracha S, Karalai C, Ponglimanont C, Tewtrakul S: Anti-allergic activity of principles from the roots and heartwood of Caesalpinia sappan on antigen-induced betahexosaminidase release. Phytotherapy research : PTR 2009, 23(7):1028-1031.

19. Li J, Cui J, Li Z, Fu X, Li J, Li H, Wang S, Zhang M: ORP8 induces apoptosis by releasing cytochrome c from mitochondria in nonsmall cell lung cancer. Oncology reports 2020, 43(5):1516-1524.

20. Liu Y, Liu H, Cao H, Song B, Zhang W, Zhang W: PBK/TOPK mediates promyelocyte proliferation via Nrf2-regulated cell cycle progression and apoptosis. Oncology reports 2015, 34(6):3288-3296.

21. Hu F, Gartenhaus RB, Eichberg D, Liu Z, Fang HB, Rapoport AP: PBK/TOPK interacts with the DBD domain of tumor suppressor p53 and modulates expression of transcriptional targets including p21. Oncogene 2010, 29(40):5464-5474.

22. Westin JR, Fayad LE: Beyond R-CHOP and the IPI in large-cell lymphoma: molecular markers as an opportunity for stratification. Current hematologic malignancy reports 2009, 4(4):218-224.

23. Dou X, Wei J, Sun A, Shao G, Childress C, Yang W, Lin Q: PBK/TOPK mediates geranylgeranylation signaling for breast cancer cell proliferation. Cancer cell international 2015, 15:27.

24. Shih MC, Chen JY, Wu YC, Jan YH, Yang BM, Lu PJ, Cheng HC, Huang MS, Yang CJ, Hsiao M et al: TOPK/PBK promotes cell migration via modulation of the PI3K/PTEN/AKT pathway and is associated with poor prognosis in lung cancer. Oncogene 2012, 31(19):2389-2400.

25. Wang WQ, Li R, Bai QX, Liu YH, Zhang WP, Wang JH, Wang Z, Li YF, Chen XQ, Huang GS: GossypolInduced Differentiation in Human Leukemia HL-60 Cells. International journal of biomedical science: IJBS 2006, 2(4):395-401.

26. Xu M, Xu S: PBK/TOPK overexpression and survival in solid tumors: A PRISMA-compliant metaanalysis. Medicine 2019, 98(10):e14766.

27. Cho SC, Choi BY: Acetylshikonin Inhibits Human Pancreatic PANC-1 Cancer Cell Proliferation by Suppressing the NF-KB Activity. Biomolecules \& therapeutics 2015, 23(5):428-433.

\section{Figures}


A

B
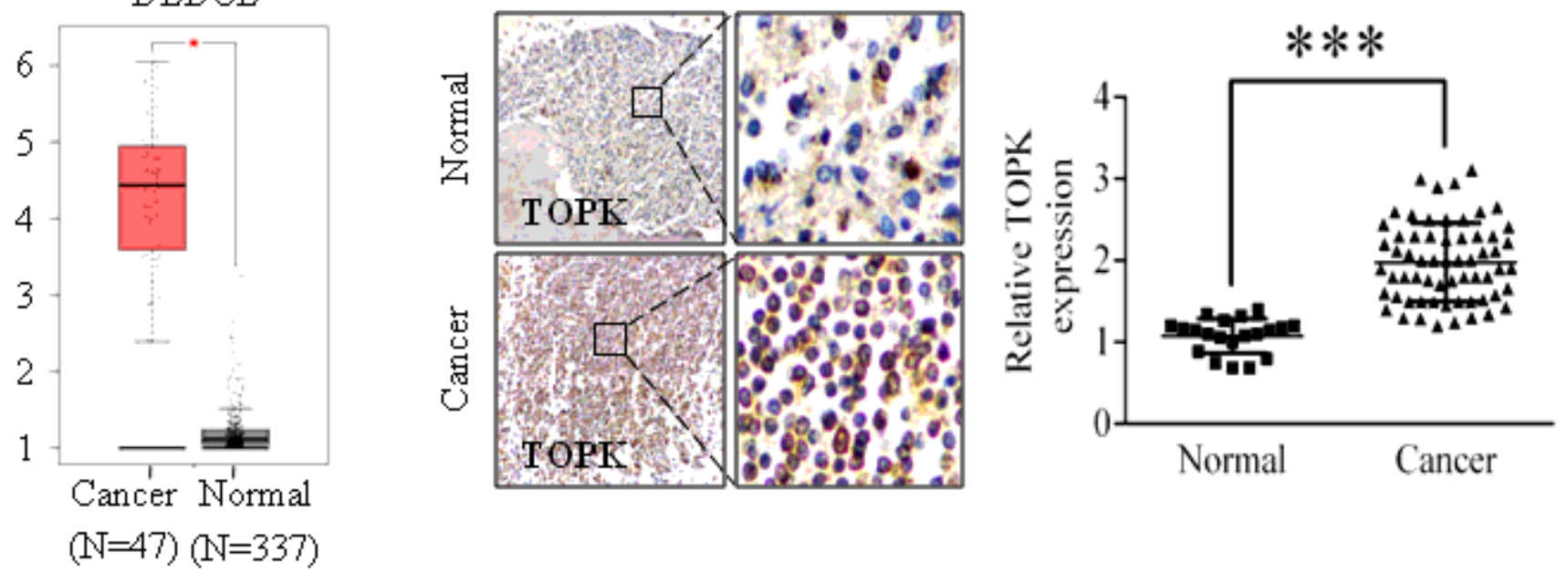

C
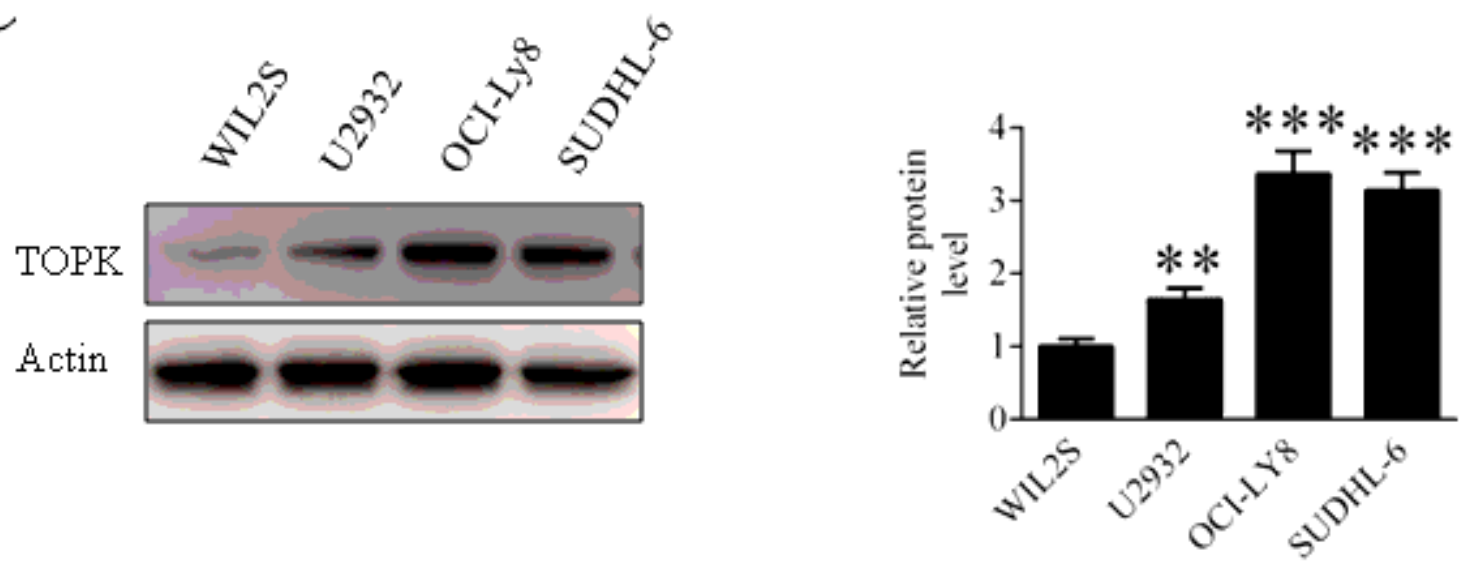

Figure 1

TOPK is highly expressed in human DLBCL. (a) TOPK expression levels in the Cancer Genome Atlas dataset in DLBCL. (b) IHC staining on DLBCL tissues array, control (20 cases), cancer (62 cases). (c) TOPK protein levels were measured in DLBCL cell lines and normal B-cell line using western blot. The data represent the mean \pm S.D. for three individual experiments $\left({ }^{\star} p<0.05,{ }^{*} p<0.01,{ }^{\star \star *} p<0.001, n=3\right)$. 

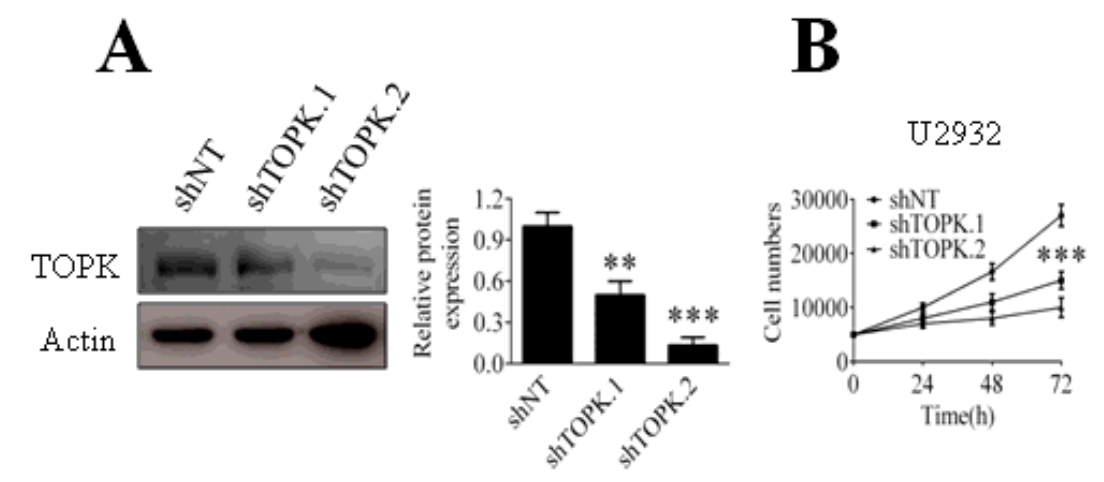

C

D
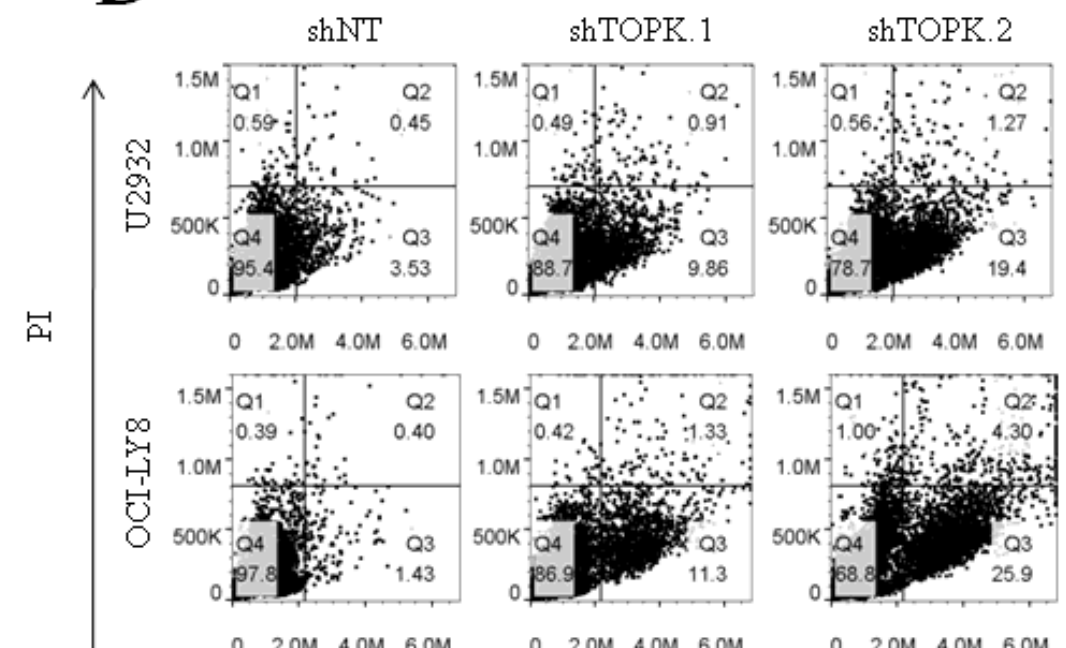

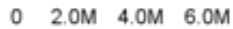

$\begin{array}{llll}0 & 2.0 \mathrm{M} \quad 4.0 \mathrm{M} \quad 6.0 \mathrm{M}\end{array}$

(0 $2.0 \mathrm{M} \quad 4.0 \mathrm{M} \quad 6.0 \mathrm{M}$
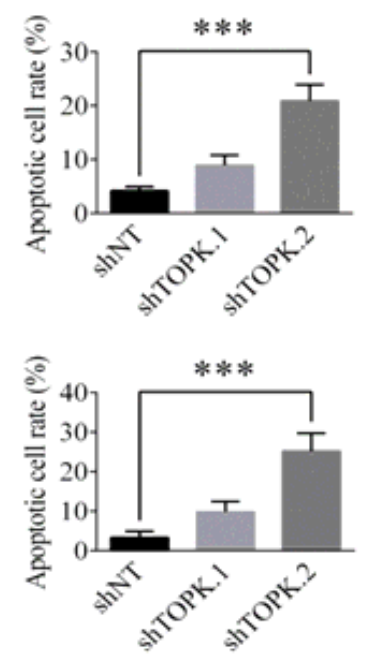

Annexin V

\section{$\mathbf{E}$}

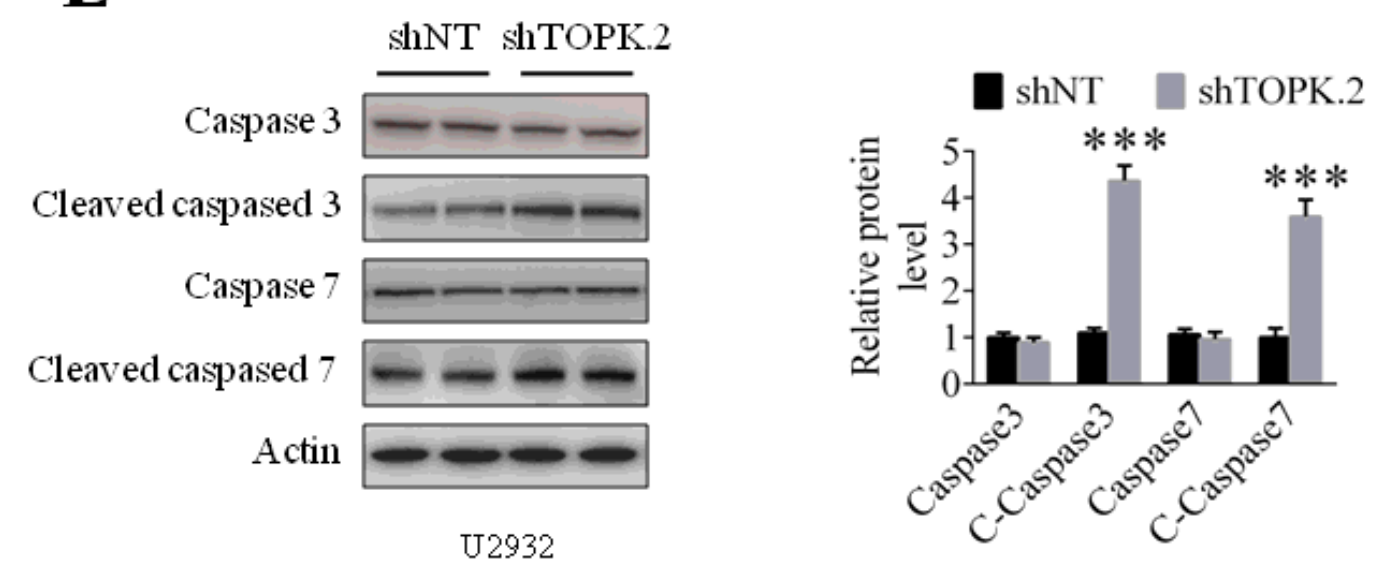

\section{Figure 2}

TOPK knockdown inhibits cell growth and induces cell apoptosis in U2932 and OCl-LY8 cell lines. (a) OClLY8 cells were infected with shTOPK.1, shTOPK.2 or shNT, the TOPK knockdown efficiency was detected by western blot. (b and c) U2932 and OCI-LY8 cells were infected with shTOPK.1, shTOPK.2 or shNT, the cell proliferation was detected at 24,48 , and $72 \mathrm{~h}$ with the MTS assay. (d) U2932 and OCI-LY8 cells were infected with shTOPK.1, shTOPK.2 or shNT, the cell apoptosis was detected staining with annexin $\mathrm{V}$ and 
PI. (e) U2932 cells were infected with shTOPK.1, shTOPK.2 or shNT, and the cleaved caspase 3 and caspase 7 protein levels were assessed with western blot. The data are the mean \pm S.D. for three individual experiments (** $\mathrm{p}<0.01,{ }^{* *} \mathrm{p}<0.001, \mathrm{n}=3$ ).
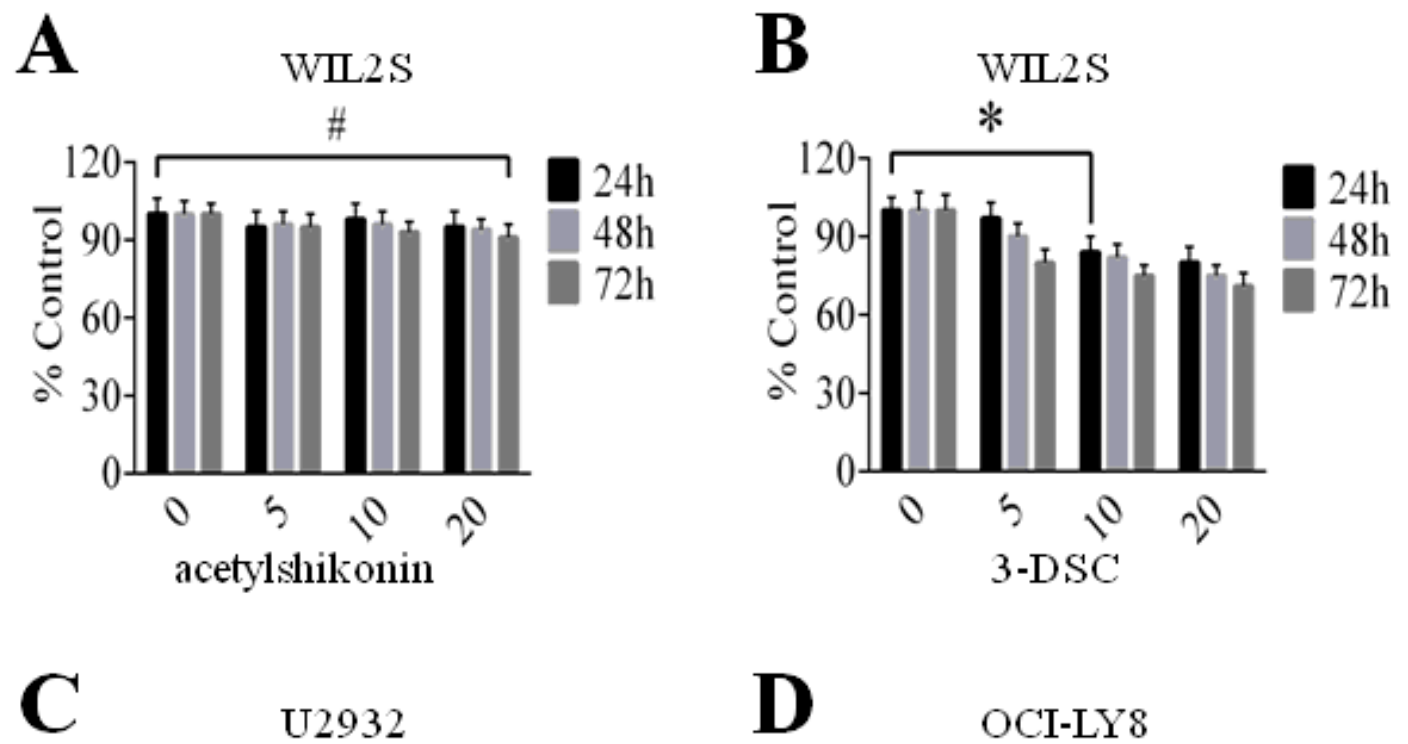

U2932

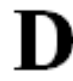

OCI-LY8
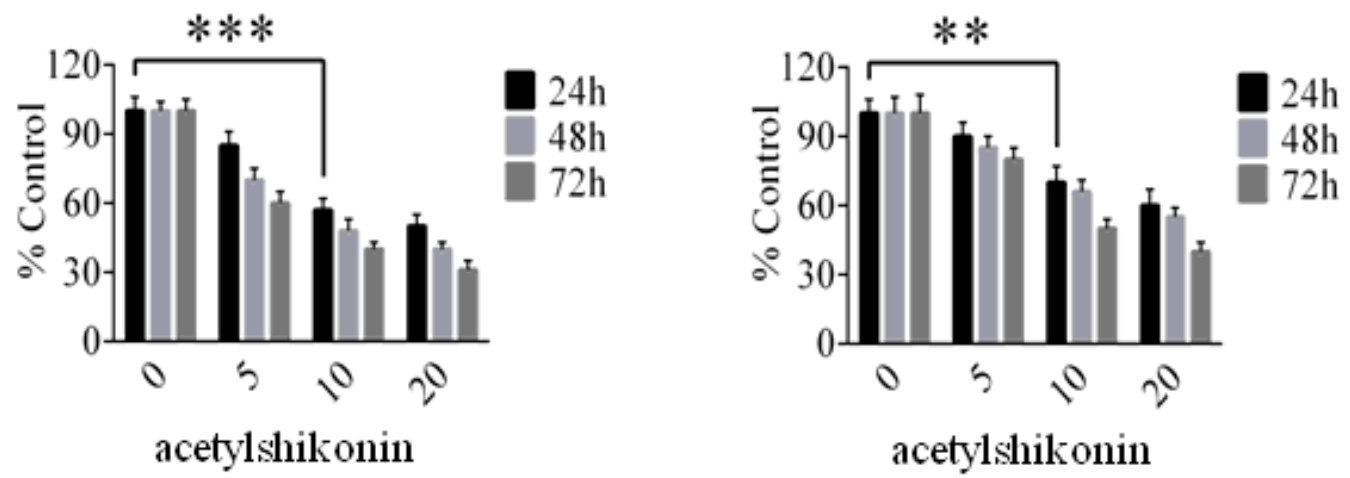

$\mathbf{E}$

U2932

F

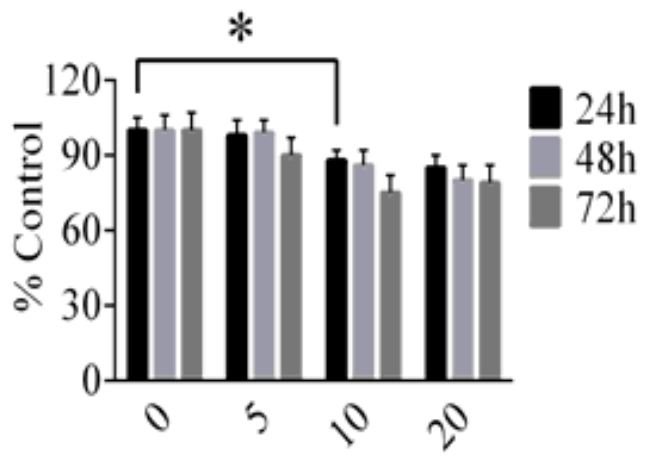

3-DSC

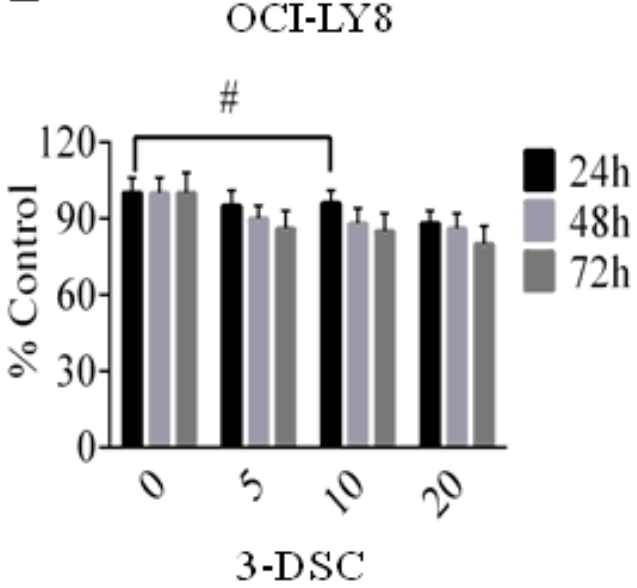

Figure 3

TOPK inhibitors suppress cell proliferation in DLBCL cells. (a-b) WIL2S cells were treated with different concentrations $(5 \mu \mathrm{M}, 10 \mu \mathrm{M}$ or $20 \mu \mathrm{M})$ of acetylshikonin or 3-DSC, cell proliferation was assessed at 24 , 
48, and $72 \mathrm{~h}$ by the MTS assay. (c-f) U2932 and OCI-LY8 cells were treated with different concentrations $(5 \mu \mathrm{M}, 10 \mu \mathrm{M}$ or $20 \mu \mathrm{M})$ of acetylshikonin or 3-DSC, cell proliferation was assessed at 24,48 , and $72 \mathrm{~h}$ by the MTS assay. (\#p $\left.>0.05,{ }^{\star} p<0.05,{ }^{* *} p<0.01,{ }^{* *} \mathrm{p}<0.001, \mathrm{n}=3\right)$.

A

DMSO

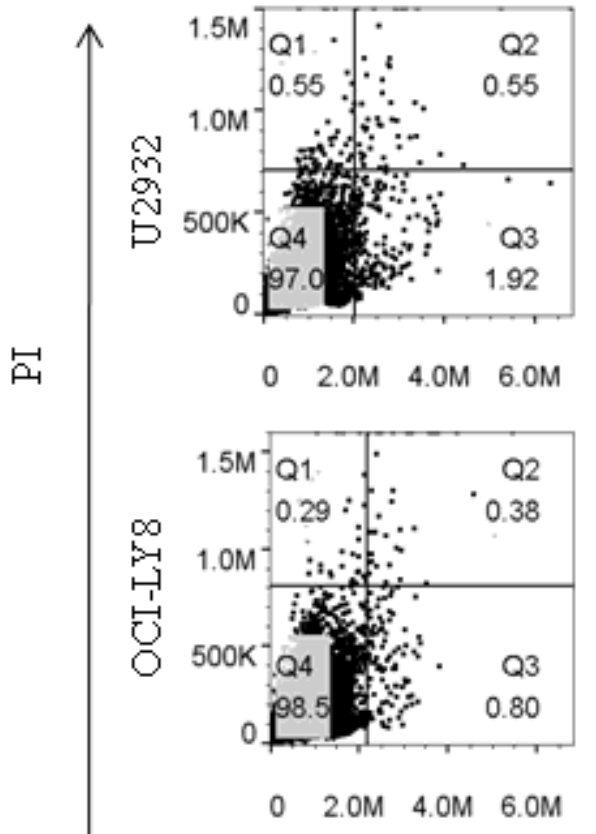

acetylshikonin
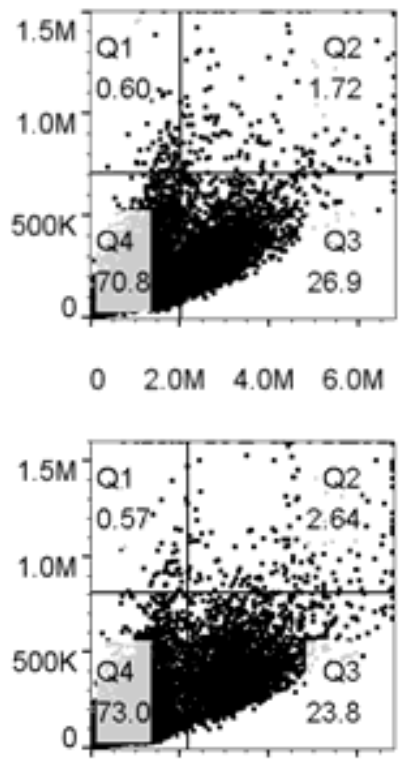

$\begin{array}{llll}0 & 2.0 \mathrm{M} & 4.0 \mathrm{M} & 6.0 \mathrm{M}\end{array}$
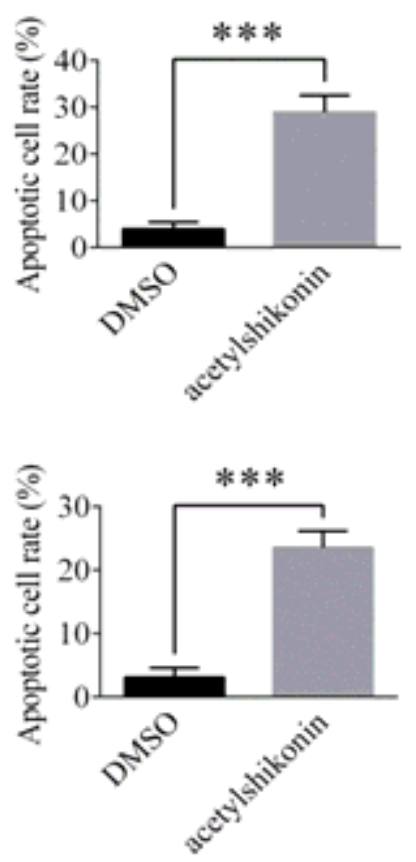

Annexin V

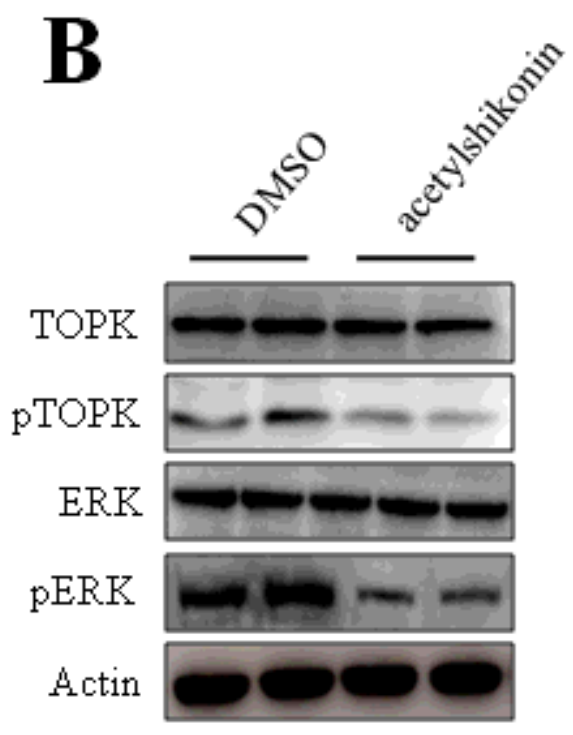

$\mathrm{U} 2932$

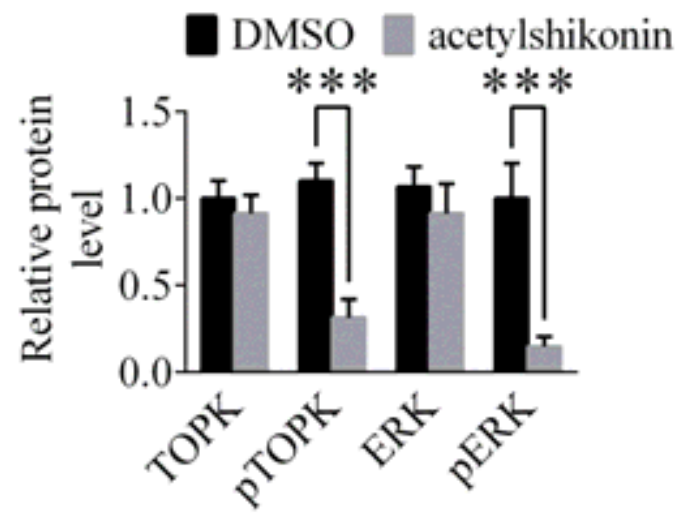

\section{Figure 4}

Acetylshikonin induces DLBCL cells apoptosis and reduces the expression of proteins involved in TOPK signaling. (a) U2932 and OCI-LY8 cells were treated with $10 \mu \mathrm{M}$ acetylshikonin for $24 \mathrm{~h}$, the cell apoptosis was detected staining with annexin V and PI. (b) U2932 cells were treated with $10 \mu \mathrm{M}$ acetylshikonin for 
24h, the protein levels of pTOPK, total TOPK, pERK, total ERK were detected by western blot. $(* \star \star p<<0.001$, $\mathrm{n}=3)$.
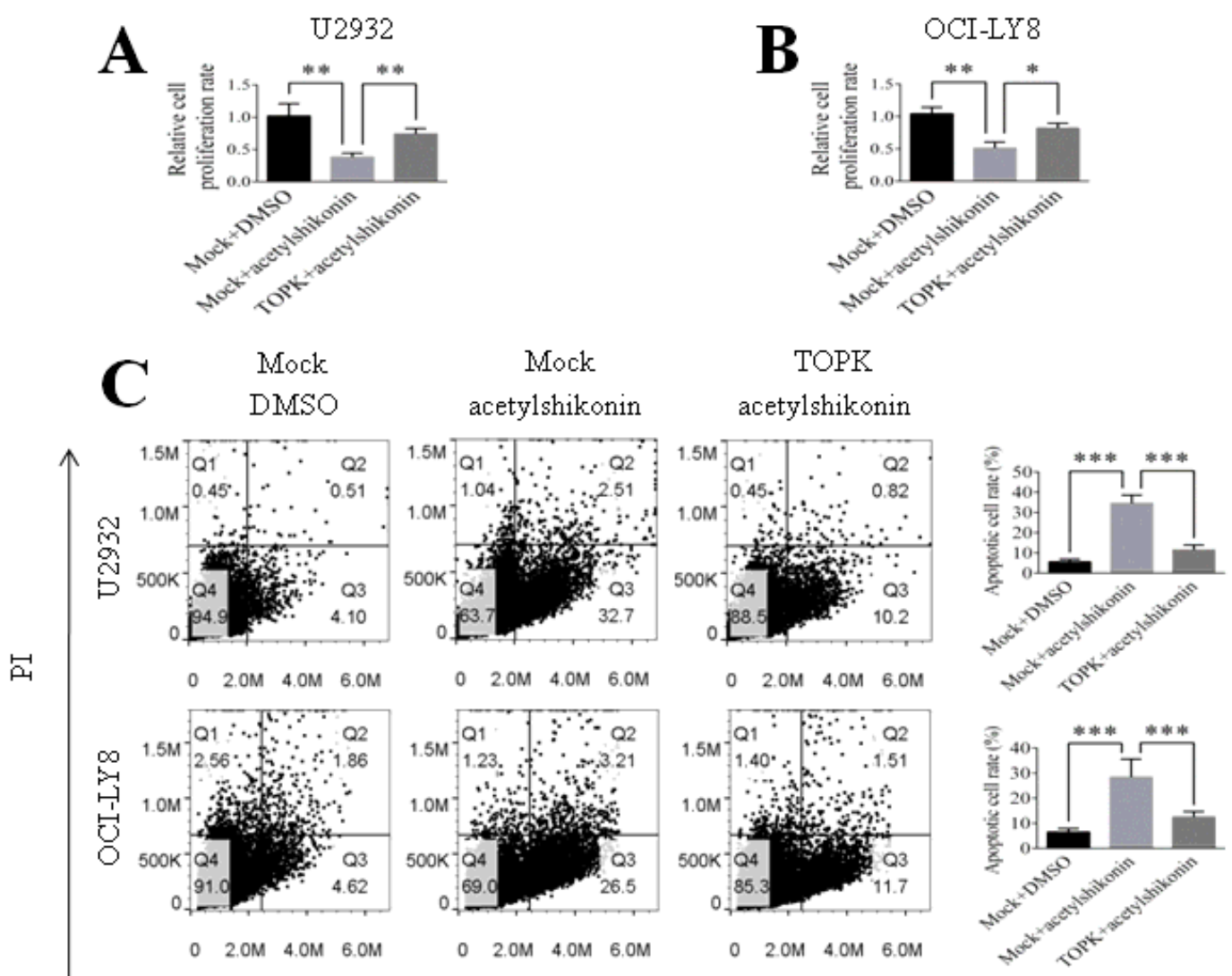

\section{Annexin V}

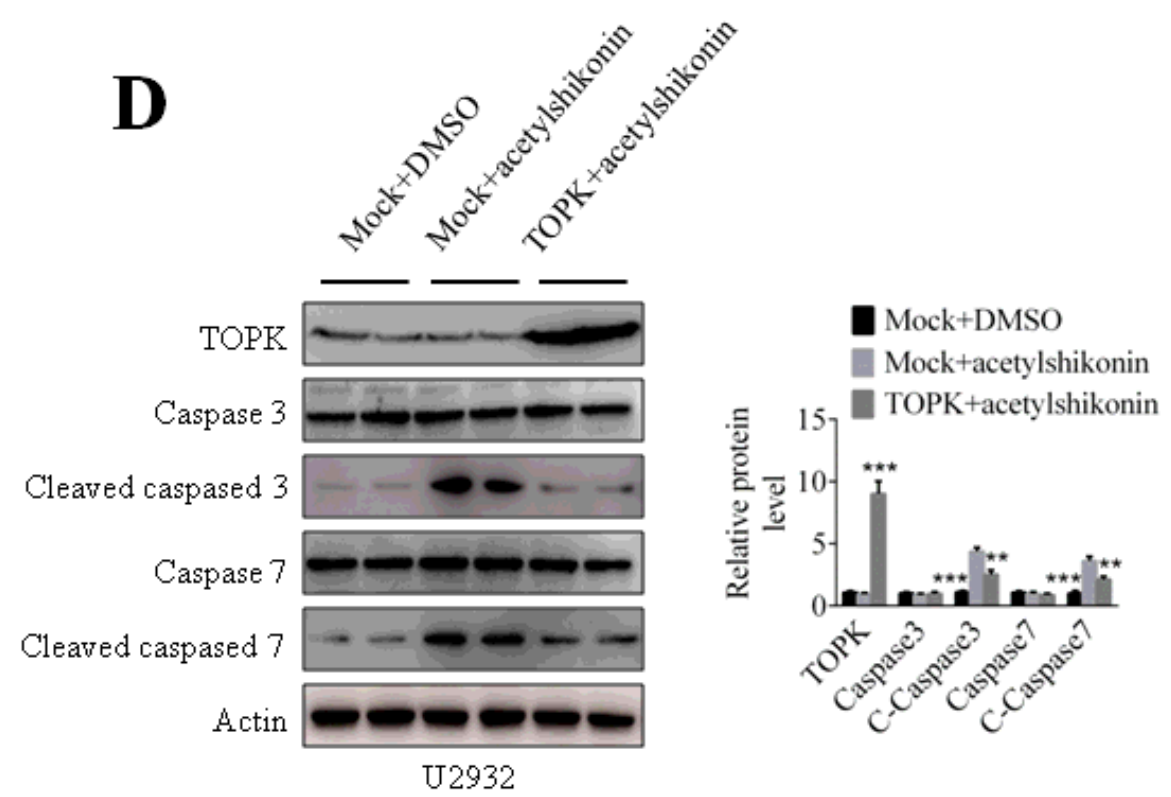

\section{Figure 5}

TOPK overexpression attenuates the effect of acetylshikonin. (a-b) U2932 and OCI-LY8 cells were infected with a lentivirus carrying TOPK cDNA or treated with $10 \mu \mathrm{M}$ acetylshikonin, cell growth was determined at $72 \mathrm{~h}$ using the MTS assay. (c) U2932 and OCI-LY8 cells were infected with a lentivirus carrying TOPK 
cDNA or treated with $10 \mu \mathrm{M}$ acetylshikonin, the cell apoptosis was detected staining with annexin $\mathrm{V}$ and PI. (d) U2932 cells were infected with a lentivirus carrying TOPK cDNA or treated with $10 \mu \mathrm{M}$ acetylshikonin, the cleaved caspase 3 and caspase 7 protein levels were assessed with western blot. ( ${ }^{*} p<$ $0.05,{ }^{* \star} \mathrm{p}<0.01,{ }^{* \star *} \mathrm{p}<0.001, \mathrm{n}=3$ ).

\section{Supplementary Files}

This is a list of supplementary files associated with this preprint. Click to download.

- SupplementFigure1.tif

- supplement.doc 